\title{
Navigation Bronchoscopy-Guided Radiofrequency Ablation for Nonsurgical Peripheral Pulmonary Tumors
}

\author{
Fangfang Xie ${ }^{a, b}$ Xiaoxuan Zheng ${ }^{a}$ Bo Xiao ${ }^{c}$ Baohui Han ${ }^{b}$ Felix J.F. Herth ${ }^{d}$ \\ Jiayuan Sun ${ }^{a, b}$
}

Departments of a Endoscopy and ${ }^{b}$ Pulmonary Medicine, Shanghai Chest Hospital, Shanghai Jiao Tong University, and ${ }^{\mathrm{C}}$ Medsphere International Co., Shanghai, PR China; ${ }^{\mathrm{d}}$ Department of Pneumology and Respiratory Care Medicine, Translational Lung Research Center Heidelberg, Member of the German Center for Lung Research, University of Heidelberg, Heidelberg, Germany

\section{Established Facts}

- Radiofrequency ablation (RFA) is a promising alternative treatment method for early-stage lung cancer in patients who are poor surgical candidates or have exhausted their maximum amount of radiation therapy. It can also be used for oligometastasis from distant cancer or as a therapy for the local control of tumors.

- RFA has been widely utilized via a percutaneous approach into a variety of organs. In the lung, percutaneous RFA is associated with severe complications, including pneumothorax, hemothorax, bronchopleural fistulas, and pleural effusions.

\section{Novel Insights}

- A bronchoscopic flexible RFA catheter can significantly decrease the severity and frequency of complications compared to percutaneous RFA.

- Navigation bronchoscopy-guided RFA is safe, efficacious, and feasible for poor surgical candidates with stage IA lung cancer or lung metastasis.

\section{Keywords}

Radiofrequency ablation · Navigation bronchoscopy · Lung cancer

\section{Abstract}

We have recently developed a flexible catheter electrode used for bronchoscopic radiofrequency ablation (RFA). Two patients with nonsurgical stage IA peripheral lung cancer

\section{KARGER}

(C) 2017 S. Karger AG, Basel

E-Mail karger@karger.com

www.karger.com/res and 1 with lung metastasis underwent treatment with flexible catheter RFA utilizing navigation bronchoscopy. Chest computed tomography (CT) and positron emission tomography/CT (PET/CT) were performed before and after RFA to assess the ablation response of the patients. One patient's tumor had no prior PET uptake and therefore no follow-up

Jiayuan Sun

Department of Endoscopy and Pulmonary Medicine Shanghai Chest Hospital, Shanghai Jiao Tong University 241 West Huaihai Road, Shanghai 200030 (PR China) E-Mail jysun1976@163.com

Felix J.F. Herth

Department of Pneumology and Critical Care Medicine Thoraxklinik University of Heidelberg

Röntgenstrasse 1, DE-69126 Heidelberg (Germany)

E-Mail felix.herth@med.uni-heidelberg.de 
PET was obtained. The first and the third patient obtained partial response to RFA, and the second patient obtained complete response 3 months after RFA. The first patient developed progressive disease 6 months after RFA. The second and the third patient achieved one-year progression-free survival. No significant complications occurred in the 3 patients. Navigation bronchoscopy-guided RFA is a safe and feasible procedure for poor surgical candidates with stage IA lung cancer or lung metastasis.

(c) 2017 S. Karger AG, Basel

\section{Introduction}

Surgical resection remains the recommended treatment approach for patients with early-stage, non-small cell lung cancer and some pulmonary metastases [1-3]. However, not all patients are eligible for surgical resection due to comorbidities or other contraindications. Stereotactic body radiotherapy and radiofrequency ablation (RFA) are recommended for patients medically inoperable as local therapy $[4,5]$. Stereotactic body radiotherapy is an effective, noninvasive treatment modality but is limited by respiratory movements, requirements for multiple treatments, cost, and the complication of radiation pneumonitis. RFA is a promising alternative treatment method with overall survival data appearing to be similar to sublobar resection and radiotherapy [6-8]. RFA has been widely used via a percutaneous method, and complications including pneumothorax, hemothorax, bronchopleural fistula, and pleural effusions are very common ranging from 15.2 to $55.6 \%$ [9]. Bronchoscopic RFA offers the chance to potentially decrease these severe complications [10]. Koizumi et al. [11] reported bronchoscopy-guided RFA to be a safe and effective procedure for medically inoperable patients. Navigational bronchoscopy techniques have allowed accurate access to peripheral pulmonary lesions for biopsy. The same techniques can be utilized for accurate placement of therapeutic tools as well as ablation catheters $[12,13]$. We present 3 cases utilizing navigational peripheral bronchoscopic RFA for ablation of tumor.

\section{Methods}

Three patients diagnosed with pulmonary tumors (2 lung tumors and 1 metastasis) by bronchoscopy underwent bronchoscopic RFA. The patients were either medically unsuitable for surgery or refused consent for thoracic surgery. Chest thin-slice computed tomography (CT) and positron emission tomography/CT
(PET/CT) were performed prior to RFA. Electromagnetic navigation bronchoscopy (superDimension/inReach system; Medtronic, Minneapolis, MN, USA) or virtual bronchoscopic navigation (VBN; DirectPath; Olympus, Tokyo, Japan) were used for navigational assistance and performed as previously described [14, 15]. Bronchoscopic RFA was performed under general anesthesia and a dispersive electrode was placed on the lower back of the patient. When the bronchoscopic peripheral catheter (extended working channel [EWC], inReach system; Medtronic, or guide sheath [GS], K-201; Olympus) reached the lesion with navigation assistance, a radial endobronchial ultrasound probe (UM-S20-20R or UM-S20-17S; Olympus) was inserted via the peripheral catheter. Combined with fluoroscopy, the radial endobronchial ultrasound probe was used to confirm the position of the EWC/GS in relation to the tumor. A flexible electrode connected to a radiofrequency generator (S500; Medsphere, Shanghai, China) (Fig. 1a) was introduced into the tumor through EWC/GS under fluoroscopic guidance.

RFA was initiated at an output power of $20 \mathrm{~W}$ with fluoroscopic monitoring and increased $5 \mathrm{~W}$ every minute until the temperature reached $100^{\circ} \mathrm{C}$. Tissue impedance and temperature were measured continuously by the generator and the output power stops automatically when the tissue impedance reaches $500 \Omega$. One to three milliliter of $2 \%$ lidocaine was injected into the lateral aperture of the electrode to alleviate the airway irritation from thermal stimulation and potentially increase the ablated area during RFA $[16,17]$. A chest CT scan was performed within $72 \mathrm{~h}$, after 1 month, and every 3 months after the procedure. PET/CT was performed 3 months after RFA to assess the treatment response. Tumor response was evaluated 3 months after the treatment using modified Response Evaluation Criteria in Solid Tumors criteria [18]. All procedures were performed by an experienced bronchoscopist (J.S.). Patients provided written informed consent for the treatment and for their medical data to be used.

There are 3 types of flexible electrodes available (Fig. 1b-e). The electrode EB- 01 is a needle electrode with the outer diameter and exposure length of 1.4 and $15 \mathrm{~mm}$, respectively. Electrodes EB-02 and EB-03 consist of a cannula with 9 deployable electrodes that open "flower-like" up to a diameter of 20 and $30 \mathrm{~mm}$, respectively, and the outer diameters of the cannula are 1.4 and $1.8 \mathrm{~mm}$, respectively.

\section{Case Report}

Case 1

A 62-year-old male was admitted with abnormal findings on chest imaging. A chest CT scan showed a $13.3 \times 11.7 \mathrm{~mm}$ lesion in the right lower lobe without any enlarged hilar or mediastinal lymph nodes. PET/CT showed hypermetabolism of the lesion with the maximal standard uptake value $\left(\mathrm{SUV}_{\max }\right)$ of 3.7. Bronchoscopy biopsy demonstrated squamous cell carcinoma with a wildtype epidermal growth factor receptor. The patient had severe dilated cardiomyopathy with a low ejection fraction and was considered medically inoperable. Electromagnetic navigation bronchoscopy-guided bronchoscopic RFA was performed. The electrode EB- 01 was used, and RFA continued 5 min with the total output power of $20 \mathrm{~W}$. Twenty-four hours after the procedure, the patient presented chest pain and fever. This resolved with conservative treatment of pain and fever. PET/CT 3 months after treatment demonstrated a reduced tumor size, and the SUV decreased 


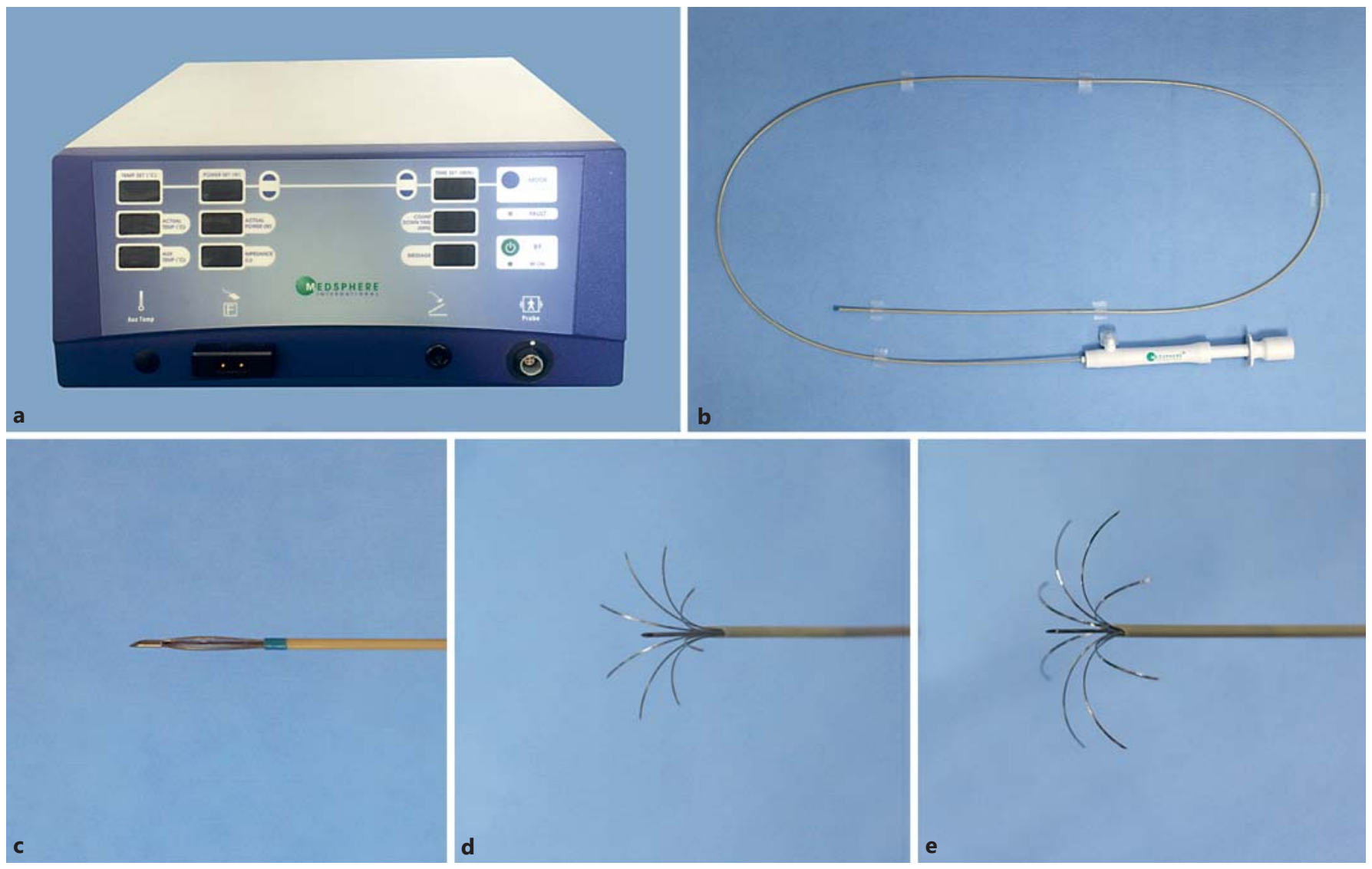

Fig. 1. Radiofrequency ablation (RFA) generator and RFA flexible electrodes. RFA generator (a); flexible electrode (b); tip of the electrode EB-01 (c); tip of the electrode EB-02 (d); tip of the electrode EB-03 (e).

to normal range $\left(\mathrm{SUV}_{\max }=1.9\right)$. The patient was classified as having obtained partial response (PR) after the treatment. Unfortunately, the lesion demonstrated progression of size on chest CT images at 6 months of follow-up. The procedure and image data of the patient are shown in Figure $2(1 \mathrm{a}-\mathrm{j})$. After progression, percutaneous RFA was performed. Six months after percutaneous RFA, the PET/CT demonstrated no fluorodeoxyglucose uptake of the tumor.

\section{Case 2}

A 65-year-old male was admitted with abnormal chest CT imaging demonstrating a $14.8 \times 10.1 \mathrm{~mm}$ nodule in the left upper lobe without hilar or mediastinal lymphadenopathy. PET-CT demonstrated hypermetabolism of the nodule with a $\mathrm{SUV}_{\max }$ of 9.5. Bronchoscopy examination revealed squamous cell carcinoma without epidermal growth factor receptor mutation. The patient had a history of prior renal transplantation and large B-cell lymphoma and refused thoracic surgery. VBN-guided bronchoscopic RFA was performed with the electrode EB-02. The electrode was inserted first into the more distal portion of the tumor and ablation with 20 $\mathrm{W}$ initiated. The ablation continued for $3 \mathrm{~min} 40 \mathrm{~s}$. The electrode was withdrawn $5 \mathrm{~mm}$ proximal, and a second ablation began. The second ablation continued $10 \mathrm{~min}$ with the output power increas- ing from 20 to $40 \mathrm{~W}$. PET/CT at 3 months revealed no uptake of the tumor. The patient obtained complete response after the treatment and is 1 year out of therapy with no evidence of disease. The procedure and image data of the patient are displayed in Figure 2 $(2 a-j)$.

\section{Case 3}

A 66-year-old male with a history of right radical nephrectomy for renal clear cell carcinoma presented to our hospital with abnormal findings on imaging. Chest CT showed a $21.8 \times 11.9 \mathrm{~mm}$ nodule in the right lower lobe without hilar or mediastinal lymph node enlargement. PET-CT showed no hypermetabolism of the nodule. Despite the negative PET, given the pre-test probability of malignancy, we proceeded with biopsy. Bronchoscopic examination revealed lung metastasis of his prior renal cell cancer. The patient declined thoracic resection, and VBN-guided bronchoscopic RFA was performed. The ablation was 6 min 40 s long using an electrode EB-02 with the output power starting at $20 \mathrm{~W}$ and increasing to $50 \mathrm{~W}$. The patient obtained PR after treatment and has been followed-up for 1 year by now with the CT abnormality almost gone on follow-up imaging. The procedure and image data of the patient are shown in Figure 3. 

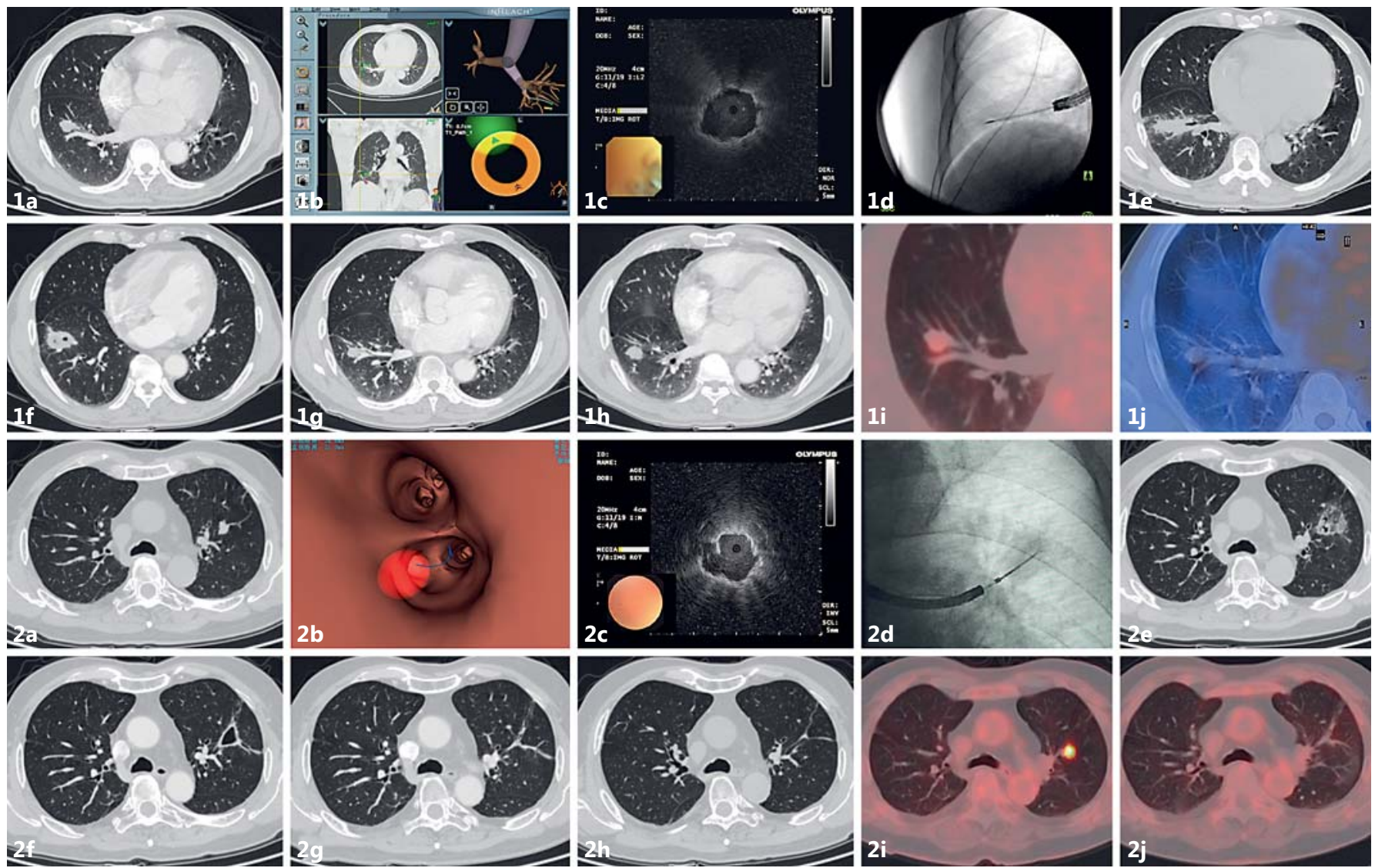

Fig. 2. 1a-j Electromagnetic navigation bronchoscopy-guided bronchoscopic radiofrequency ablation (RFA) of Case 1. Chest CT before RFA (a); real-time electromagnetic navigation screen of the sensor probe nearest to the tumor (b); radial ultrasound probe image of the tumor (c); bronchoscopic RFA with the electrode EB-01 under the guidance of fluoroscopy (d); chest CT within $72 \mathrm{~h}$ after RFA showing that the ablation zone appeared larger than the original tumor (e); chest CT 1 month after RFA, showing a cavity in the ablation site (f); chest CT 3 months after RFA showing that the tumor decreased in size (g); chest CT 6 months after RFA showing progression (h); preoperative PET/CT (maximum standardized uptake value [SUV $\mathrm{SUx}_{\max }$ ] 3.7) (i); PET/CT 3 months after RFA (SU-
$\mathrm{V}_{\max }$ 1.9) (j). 2a-j Virtual bronchoscopic navigation-guided bronchoscopic RFA of Case 2. Chest CT before RFA (a); virtual bronchoscopic navigation screen of the pathway (b); radial ultrasound probe image of the tumor (c); bronchoscopic RFA with the electrode EB-02 under the guidance of fluoroscopy (d); chest CT within $72 \mathrm{~h}$ after RFA, showing partial emptying of the ablation zone (e); chest CT 1 month after RFA, demonstrating a cavity (f); chest CT 3 months after RFA, showing linear lines and a scarring shadow (g); chest CT 1 year after RFA with a minimal residual scar (h); preoperative PET/CT (SUV $\max 9.5$ ) (i); PET/CT 3 months after RFA, showing no fluorodeoxyglucose uptake (j).

\section{Discussion}

RFA holds the promise of a potentially effective treatment for patients with early-stage lung cancer or lung metastasis who are not surgical candidates or decline surgical intervention $[19,20]$. The 3 flexible electrodes were developed for bronchoscopic RFA and are applicable for tumor sizes up to $30 \mathrm{~mm}$. The electrode EB-01, which was suitable for a lesion $<15.0 \mathrm{~mm}$, was used in Case 1. Though the tumor decreased in size at 3 months after broncho- scopic RFA, there was an increase at 6 months. It is unclear if this is a direct failure of RFA, a choice of too small of a catheter, or limitations to peripheral bronchoscopy (i.e., we were tangential or not directly central within the tumor). We selected EB-02 for Case 2 as it has a larger area of energy application. We also did 2 ablations of the tumor along the long axis. Of note, this tumor had a complete response after the treatment. As Case 3 had no prior fluorodeoxyglucose uptake, we did not have the benefit of PET to follow tumor response. Patient 3 obtained a PR 

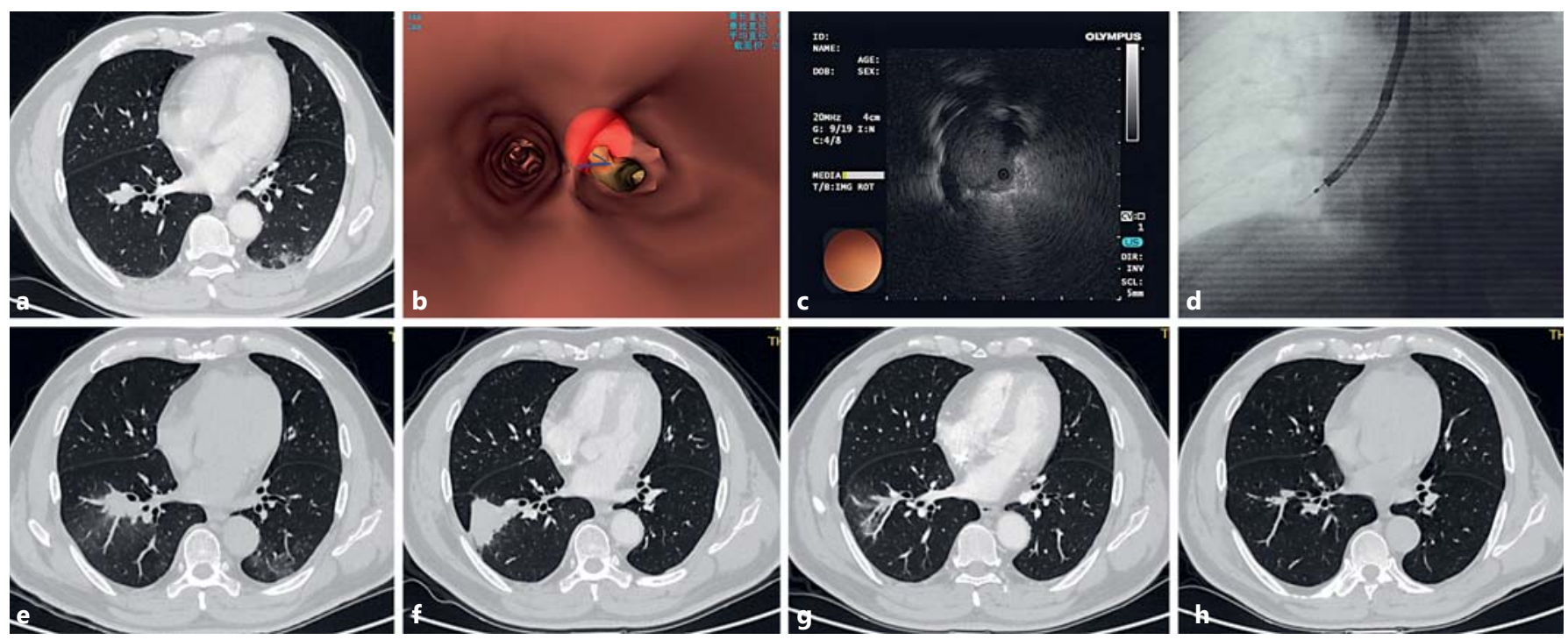

Fig. 3. Virtual bronchoscopic navigation-guided bronchoscopic radiofrequency ablation (RFA) of Case 3. Chest CT before RFA (a); virtual bronchoscopic navigation screen of the pathway (b); radial ultrasound probe image of the tumor (c); bronchoscopic RFA with the electrode EB-02 under the guidance of fluoroscopy (d); chest CT within $72 \mathrm{~h}$ after RFA showing that the ablation zone

appeared larger than the original tumor (e); chest CT 1 month after RFA showing that the ablation zone continued to increase (f); chest CT 3 months after RFA showing that the ablation zone became smaller (g); chest CT 1 year after RFA showing that the ablation zone demonstrated linear scar lines (h).

but has been followed-up for 1 year with near resolution of the radiographic abnormality. The small size limits the re-biopsy potential of the lesion and it will be followed radiographically. As this is early data on a new technology, we are continuing to learn and adapt our techniques as the clinical scenario dictates.

Traditionally, RFA is performed percutaneously. This places obvious limits for the routine use in the lung. With a high incidence rate of complications such as pneumothorax, hemothorax, and bronchopleural fistulas, there has been appropriate concern $[6,9,21,22]$. In addition to complications, some locations are anatomically difficult to reach and also carry the risk of pleural metastasis/direct spread of the tumor [23]. Bronchoscopic RFA offers the opportunity to reach difficult locations and possibly reduce the occurrence of RFA pulmonary complications. Though our sample size was small, we observed no severe complications in the 3 patients. Our study showed that bronchoscopic RFA was a safe procedure, as had been reported previously [11]. We speculate that bronchoscopic RFA has other potentially significant advantages. First, as the radiofrequency electrode was introduced into the tumor through the bronchus, the vaporization of tissue and necrosis induced by bronchoscopic RFA can directly communicate with the bronchus reducing the occurrence

Bronchoscopic RFA for Pulmonary Tumors 


\section{Acknowledgment}

The study was supported by Shanghai Municipal Hospitals' Rising and Leading Technology Program (SHDC12015115), Scientific Research Program by Science and Technology Commission of Shanghai Municipality (15441900502, 15411964300, 16441900702, 16441903502).

\section{Financial Disclosure and Conflicts of Interest}

The authors have no conflicts of interest to disclose.

\section{References}

1 Scott WJ, Howington J, Feigenberg S, Movsas B, Pisters K: Treatment of non-small cell lung cancer stage I and stage II: ACCP evidencebased clinical practice guidelines (ed 2). Chest 2007;132(3 suppl):234s-242s.

2 Erhunmwunsee L, D’Amico TA: Surgical management of pulmonary metastases. Ann Thorac Surg 2009;88:2052-2060.

3 Friedel G, Pastorino U, Buyse M, Ginsberg RJ, Girard P, Goldstraw P, Johnston M, McCormack P, Pass H, Putnam JB, Toomes H: Resection of lung metastases: long-term results and prognostic analysis based on 5206 cases - the International Registry of Lung Metastases (in German). Zentralbl Chir 1999;124:96103.

4 Gould MK, Donington J, Lynch WR, Mazzone PJ, Midthun DE, Naidich DP, Wiener RS: Evaluation of individuals with pulmonary nodules: when is it lung cancer? Diagnosis and management of lung cancer, ed 3: American College of Chest Physicians evidencebased clinical practice guidelines. Chest 2013; 143(5 suppl):e93S-e120S.

5 Harzheim D, Eberhardt R, Hoffmann $\mathrm{H}$, Herth FJ: The solitary pulmonary nodule. Respiration 2015;90:160-172.

6 Bilal H, Mahmood S, Rajashanker B, Shah R: Is radiofrequency ablation more effective than stereotactic ablative radiotherapy in patients with early stage medically inoperable non-small cell lung cancer? Interact Cardiovasc Thorac Surg 2012;15:258-265.

7 Dupuy DE, Fernando HC, Hillman S, Ng T, Tan AD, Sharma A, Rilling WS, Hong K, Putnam JB: Radiofrequency ablation of stage IA non-small cell lung cancer in medically inoperable patients: results from the American College of Surgeons Oncology Group Z4033 (Alliance) trial. Cancer 2015;121:3491-3498.
8 Safi S, Rauch G, op den Winkel J, Kunz J, Schneider T, Bischof M, Heussel CP, Huber PE, Herth FJ, Dienemann H, Hoffmann H: Sublobar resection, radiofrequency ablation or radiotherapy in stage I non-small cell lung cancer. Respiration 2015;89:550-557.

9 Zhu JC, Yan TD, Morris DL: A systematic review of radiofrequency ablation for lung tumors. Ann Surg Oncol 2008;15:1765-1774.

10 Harris K, Puchalski J, Sterman D: Recent advances in bronchoscopic treatment of peripheral lung cancers. Chest 2017;151:674-685.

11 Koizumi T, Tsushima K, Tanabe T, Agatsuma T, Yokoyama T, Ito M, Kanda S, Kobayashi T, Yasuo M: Bronchoscopy-guided cooled radiofrequency ablation as a novel intervention therapy for peripheral lung cancer. Respiration 2015;90:47-55.

12 Anantham D, Feller-Kopman D, Shanmugham LN, Berman SM, DeCamp MM, Gangadharan SP, Eberhardt R, Herth F, Ernst A: Electromagnetic navigation bronchoscopy-guided fiducial placement for robotic stereotactic radiosurgery of lung tumors: a feasibility study. Chest 2007;132:930-935.

13 Harms W, Krempien R, Grehn C, Hensley F, Debus J, Becker HD: Electromagnetically navigated brachytherapy as a new treatment option for peripheral pulmonary tumors. Strahlenther Onkol 2006;182:108-111.

14 Sun J, Mao X, Xie F, Han B, Chen H: Electromagnetic navigation bronchoscopy guided injection of methylene blue combined with hookwire for preoperative localization of small pulmonary lesions in thoracoscopic surgery. J Thorac Dis 2015;7:E652-E656.

15 Asano F, Matsuno Y, Tsuzuku A, Anzai M, Shinagawa N, Yamazaki K, Ishida T, Moriya $\mathrm{H}$ : Diagnosis of peripheral pulmonary lesions using a bronchoscope insertion guidance system combined with endobronchial ultrasonography with a guide sheath. Lung Cancer 2008;60:366-373.
16 Provenzano DA, Lassila HC, Somers D: The effect of fluid injection on lesion size during radiofrequency treatment. Reg Anesth Pain Med 2010;35:338-342.

17 Goldberg SN, Ahmed M, Gazelle GS, Kruskal JB, Huertas JC, Halpern EF, Oliver BS, Lenkinski RE: Radio-frequency thermal ablation with $\mathrm{NaCl}$ solution injection: effect of electrical conductivity on tissue heating and coagulation-phantom and porcine liver study. Radiology 2001;219:157-165.

18 Herrera LJ, Fernando HC, Perry Y, Gooding WE, Buenaventura PO, Christie NA, Luketich JD: Radiofrequency ablation of pulmonary malignant tumors in nonsurgical candidates. J Thorac Cardiovasc Surg 2003;125:929-937.

19 Lencioni R, Crocetti L, Cioni R, Suh R, Glenn D, Regge D, Helmberger T, Gillams AR, Frilling A, Ambrogi M, Bartolozzi C, Mussi A: Response to radiofrequency ablation of pulmonary tumours: a prospective, intention-totreat, multicentre clinical trial (the RAPTURE study). Lancet Oncol 2008;9:621-628.

20 Eberhardt R, Kahn N, Herth FJ: "Heat and destroy": bronchoscopic-guided therapy of peripheral lung lesions. Respiration 2010;79: 265-273.

21 Lanuti M, Sharma A, Digumarthy SR, Wright CD, Donahue DM, Wain JC, Mathisen DJ, Shepard JA: Radiofrequency ablation for treatment of medically inoperable stage I non-small cell lung cancer. J Thorac Cardiovasc Surg 2009;137:160-166.

22 Cooper R, Bann D, Wloch EG, Adams JE, Kuh D: CT-guided needle biopsy of lung lesions: a survey of severe complication based on 9783 biopsies in Japan. Eur J Radiol 2006;59:60-64.

23 Schreiner AM, Jones JG, Swistel AJ, Hoda RS: Transthoracic fine needle aspiration resulting in implantation metastasis in the superficial tissues of the breast. Cytopathology 2013;24: 58-60. 